\title{
Conservative flight with a varying load factor and closed form solutions to the aircraft motion - A technical note
}

\author{
S N MAITRA
}

Flat 303, Elite Galaxy, Ramnagar Colony, National Defence Academy (NDA) Pashan Road, Bavdhan, Pune 411021

e-mail: soumen_maitra@yahoo.co.in

MS received 22 May 2009; revised 24 June 2010; accepted 30 September 2010

\begin{abstract}
Conservative flight performance of an aircraft with constant load factor was analysed by Miele. In this paper, conservative flight with a varying load factor is considered. The load factor being a control parameter is varied in such a way that it gives rise to closed-form solutions to aircraft motion. With this typical load factor function the geometry of the trajectory has been found to be a loop as is with Miele's analysis. Finally, the range and endurance have been determined for two different constant parameters associated with the load factor function.
\end{abstract}

Keywords. Conservative flight; varying load factor; aircraft motion; aircraft performance.

\section{Introduction}

A flight path along which the thrust is kept equal to the drag at all time instants is called a conservative path. Miele (1962) considered conservative flight of an aircraft in a vertical plane over a flat Earth. Within the frame work of flat earth hypotheses the equations of motion of an aircraft as obtained by Miele is

$$
\begin{aligned}
& \dot{X}-V \cos \gamma=0 \\
& \dot{h}-V \sin \gamma=0 \\
& T-D-W\left(\sin \gamma+\frac{\dot{V}}{g}\right)=0 \\
& L-W\left(\cos \gamma+\frac{V \dot{\gamma}}{g}\right)=0 \\
& \dot{W}+c T=0 \\
& L=\frac{1}{2} C_{L} P S V^{2},
\end{aligned}
$$


where $X$ is the horizontal distance, $h$ the altitude, $V$ the velocity, $\gamma$ the path inclination to the horizontal, $W$ the weight, $D$ the drag, $L$ the left, $T$ the thrust, $c$ the specific fuel consumption, $g$ the acceleration due to gravity, and the dot sign a derivative with respect to time $t$.

While the thrust is always equal to the drag and load factor is defined as the lift-to-weight ratio $\left(n=\frac{L}{W}\right)$, eq. (1) of motion can be written as

$$
\begin{aligned}
& \dot{X}-V \cos \gamma=0 \\
& \dot{h}-V \sin \gamma=0 \\
& \dot{V}+g \sin \gamma=0 \\
& \dot{\gamma}-\frac{g}{V}(n-\cos \gamma)=0 .
\end{aligned}
$$

By introducing the dimensionless co-ordinates

$$
\xi=\frac{X g}{V_{i}^{2}}, \quad \eta=\frac{h g}{V_{i}^{2}}, \quad u=\frac{V}{V_{i}},
$$

where $V_{i}$ is the initial velocity; eliminating the time $t$ and selecting the path inclination as the new independent variable, set (2) can be transformed to the form

$$
\begin{aligned}
& \frac{d \xi}{d \gamma}=\frac{u^{2} \cos \gamma}{n-\cos \gamma} \\
& \frac{d \eta}{d \gamma}=\frac{u^{2} \sin \gamma}{n-\cos \gamma} \\
& \frac{d u}{d \gamma}=-\frac{u \sin \gamma}{n-\cos \gamma} .
\end{aligned}
$$

Miele analysed the performance of an aircraft in conservative flight taking constant load factor (n) and gave complete solutions of eq. (3). The load factor, being a control parameter related to an aircraft or a space craft flying in atmosphere, can be varied or kept constant as per needs from an engineering point of view.

In the present technique the variation of the load factor is manipulated by an aircraft pilot according to such a rule that yields closed-form solutions to the differential eq. (3). For this purpose the load factor can be devised as the following function of the path inclination without loss of physical and engineering significance

$$
n=b n_{0}-(b-1) \cos \gamma, \quad b \neq 1, \quad 0 \leq \gamma \leq \pi,
$$

where $n_{0}$ and $b$ are constant parameters specific to an aircraft in conservative flight.

Substitution of eqs. (4) in (3) yields

$$
\begin{aligned}
\frac{d \xi}{d \gamma} & =\frac{u^{2} \cos \gamma}{b\left(n_{0}-\cos \gamma\right)} \\
\frac{d \eta}{d \gamma} & =\frac{u^{2} \sin \gamma}{b\left(n_{0}-\cos \gamma\right)} \\
\frac{d u}{d \gamma} & =\frac{-u \sin \gamma}{b\left(n_{0}-\cos \gamma\right)} .
\end{aligned}
$$




\section{Integral performance}

In order to solve the differential eq. (5) the following initial conditions are assumed:

$$
\gamma_{i}=\xi_{i}=\eta_{i}=0, \quad u_{i}=1,
$$

as a result of which, the integration of the third equation becomes

$$
u=\left(\frac{n_{0}-1}{n_{0}-\cos \gamma}\right)^{1 / b} .
$$

This function gives the velocity distribution along the conservative path. The velocity at the highest point $(\gamma=\pi)$ of the path is

$$
u=\left(\frac{n_{0}-1}{n_{0}+1}\right)^{1 / b},
$$

whereas the load factors at the lowest and highest points are

$$
\begin{gathered}
n_{i}=b n_{0}-b+1 \\
n_{\pi}=b n_{0}+b-1 .
\end{gathered}
$$

The load factor at the vertical position is

$$
n_{\pi / 2}=b n_{0} .
$$

Thus the constant parameters $a$ and $b$ are evaluated from (9) as

$$
\begin{aligned}
b & =\frac{n_{\pi}-n_{i}+2}{2} . \\
n_{0} & =1+\frac{2\left(n_{i}-1\right)}{n_{\pi}-n_{i}+2} .
\end{aligned}
$$

For $n_{i}>1$ and $b>0, n_{0}>1$ and $n>1$; these are to be satisfied in the present design.

To determine the geometry of the trajectory, relationship (7) is employed in the first of set (5) so that

$$
\frac{d \xi}{d \gamma}=\frac{\left(n_{0}-1\right)^{2 / b}}{b\left(n_{0}-\cos \gamma\right)^{b+2 / b}}
$$

Owing to the initial consitions (6), the horizontal distance becomes

$$
\xi=\frac{\left(n_{0}-1\right)^{2 / b}}{b} \int_{0}^{\gamma} \frac{\cos \theta d \theta}{\left(n_{0}-\cos \theta\right)^{b+2 / b}} .
$$

The evaluation of this integral depends on the value of $b$. For example, $b=2, \frac{1}{2}$ give closedform integral; otherwise the expression for the distance can be put as a summation of a series due to Binomial expansion (for $\frac{\cos \gamma}{n_{0}}<1$ ):

$$
\xi=\frac{\left(n_{0}-1\right)^{2 / b}}{b n_{0}^{p}}\left[I_{1}+\sum_{r=1}^{\infty} \frac{p(p+1) \cdots(p+r+1)}{n_{0}^{r} \cdot \angle r} I_{r+1}\right],
$$


where

$$
\begin{aligned}
p & =\frac{b+2}{b} \\
I_{1} & =\int_{0}^{\gamma} \cos \theta d \theta=\sin \gamma \\
I_{r+1} & =\int_{0}^{\gamma} \cos ^{r+1} \theta d \theta .
\end{aligned}
$$

Using (6) and (7) in the second of eq. (5), the altitude distribution is acquired:

$$
\eta=\frac{1}{2}\left[1-\left(\frac{n_{0}-1}{n_{0}-\cos \gamma}\right)^{2 / b}\right]
$$

The highest altitude is

$$
\eta_{f}=\frac{1}{2}\left[1-\left(\frac{n_{0}-1}{n_{0}+1}\right)^{2 / b}\right] .
$$

Thus eqs. (15) and (17) supply the geometry of the trajectory in parametric form having the path inclination as the parameter. The time-path-inclination distribution is due to eqs. (6), (7), (4) and the last eq. of (2) so that

$$
t=\frac{V_{i}\left(n_{0}-1\right)^{1 / b}}{g b} \int_{0}^{\gamma} \frac{d \theta}{\left(n_{0}-\cos \theta\right)^{(b+1) / b}} .
$$

The possibility of this integration by the fundamental theorem of integral calculus depends on the value of $b$. However, the same can be computed in series as in the case of horizontal distance:

$$
t=\frac{V_{i}\left(n_{0}-1\right)^{1 / b}}{g b n_{0}^{q}}\left[r+\sum_{r=1}^{\infty} \frac{q(q+1) \cdots(q+r+1)}{n_{o}^{r} / r} I_{r}\right],
$$

where $q=\frac{b+1}{b}$.

\section{Range and endurance}

In this section, the range $\xi_{f}$ and endurance $t_{f}$ are calculated for $b=2$ and $b=\frac{1}{2}$ respectively.

\subsection{Case I. $b=2$}

Here in view of eqs. (14) and (17), the co-ordinates of any point of the loop trajectory are represented by

$$
\begin{aligned}
\xi & =\frac{n_{0}-1}{2} \int_{0}^{\gamma} \frac{\cos \theta d \theta}{\left(n_{0}-\cos \theta\right)^{2}} \\
& =\frac{1}{n_{0}+1}\left[\frac{n_{0} \sin \gamma}{2\left(n_{0}-\cos \gamma\right)}+\frac{1}{\sqrt{n_{0}^{2}-1}} \tan ^{-1}\left(\sqrt{\frac{n_{0}+1}{n_{0}-1}} \tan \frac{\gamma}{2}\right)\right] .
\end{aligned}
$$




$$
\eta=\frac{1}{2}\left[1-\frac{n_{0}-1}{n_{0}-\cos \gamma}\right] .
$$

Now the co-ordinates of the highest point $(\gamma=\pi)$ of the loop are

$$
\begin{aligned}
\xi_{\pi} & =\frac{\pi}{2\left(n_{0}+1\right) \sqrt{n_{0}^{2}-1}} . \\
\eta_{\pi} & =\frac{1}{n_{0}+1} .
\end{aligned}
$$

The range is

$$
\begin{aligned}
\xi_{f} & =\frac{n_{0}-1}{2} \int_{0}^{2 \pi} \frac{\cos \theta d \theta}{\left(n_{0}-\cos \theta\right)^{2}} \\
& =\left(n_{0}-1\right) \int_{0}^{\pi} \frac{\cos \theta d \theta}{\left(n_{0}-\cos \theta\right)^{2}} \quad(\because \cos (2 \pi-\theta)=\cos \theta) \\
& =\frac{\pi}{\left(n_{0}+1\right) \sqrt{n_{0}^{2}-1}} \\
t_{f} & =\frac{V_{i}(n-1)^{1 / 2}}{g} \int_{0}^{\pi} \frac{d \theta}{\left(n_{0}-\cos \theta\right)^{3 / 2}} .
\end{aligned}
$$

\subsection{Case II. $b=\frac{1}{2}$}

In this case using the same equations as in the previous case, the parametric equations of the trajectory can be derived as

$$
\begin{aligned}
\xi & =2\left(n_{0}-1\right)^{4} \int_{0}^{\gamma} \frac{\cos \theta d \theta}{\left(n_{0}-\cos \theta\right)^{5}} \\
& =2\left(n_{0}-1\right)^{4}\left[n_{0} I_{5}-I_{4}\right],
\end{aligned}
$$

where

$$
\begin{aligned}
& I_{5}=\int_{0}^{\gamma} \frac{d \theta}{\left(n_{0}-\cos \gamma\right)^{5}} \\
& I_{4}=\int_{0}^{\gamma} \frac{d \theta}{\left(n_{0}-\cos \theta\right)^{4}} .
\end{aligned}
$$

On integration by reduction process the following relationships are established

$$
\begin{aligned}
& I_{5}=\frac{1}{4\left(n_{0}^{2}-1\right)}\left\{\frac{\sin \gamma}{\left(n_{0}-\cos \gamma\right)^{4}}+7 n_{0} I_{4}-3 I_{3}\right\} . \\
& I_{4}=\frac{1}{3\left(n_{0}^{2}-1\right)}\left\{\frac{\sin \gamma}{\left(n_{0}-\cos \gamma\right)^{3}}+5 n_{0} I_{3}-2 I_{2}\right\}
\end{aligned}
$$




$$
\begin{aligned}
& I_{3}=\frac{1}{2\left(n_{0}^{2}-1\right)}\left\{\frac{\sin \gamma}{\left(n_{0}-\cos \gamma\right)^{2}}+3 n_{0} I_{2}-I_{1}\right\} \\
& I_{2}=\frac{1}{n_{0}^{2}-1}\left\{\frac{\sin \gamma}{n_{0}-\cos \gamma}+n_{0} I_{1}\right\} \\
& I_{1}=\int_{0}^{\gamma} \frac{d \theta}{n_{0}-\cos \theta}=\frac{2}{\sqrt{n_{0}^{2}-1}} \tan ^{-1}\left(\tan \frac{\gamma}{2} \sqrt{\frac{n_{0}+1}{n_{0}-1}}\right) .
\end{aligned}
$$

Eliminating $I_{i}(i=1,2, \ldots, 5)$ from eqs. (25), (26) and (27), the horizontal distance $\xi$ can be found out:

Let $J_{i}=\left[I_{i}\right]_{0}^{\pi}(i=1,2, \ldots, 5)$. Then eq. (27) yield

$$
\begin{aligned}
& J_{1}=\int_{0}^{\pi} \frac{d \gamma}{n_{0}-\cos \gamma}=\frac{n_{0} \pi}{\sqrt{n_{0}^{2}-1}} \\
& J_{2}=\frac{J_{1} n_{0}}{n_{0}^{2}-1}=\frac{n_{0} \pi}{\left(n_{0}^{2}-1\right) \sqrt{n_{0}^{2}-1}} \\
& J_{3}=\frac{3 n_{0} J_{2}-J_{1}}{2\left(n_{0}^{2}-1\right)}=\frac{2 n_{0}^{2}+1}{2\left(n_{0}^{2}-1\right)^{2}} \cdot \frac{\pi}{\sqrt{n_{0}^{2}-1}} \\
& J_{4}=\frac{5 n_{0} J_{3}-2 J_{2}}{3\left(n_{0}^{2}-1\right)}=\frac{n_{0} \pi\left(2 n_{0}^{2}+3\right)}{2\left(n_{0}^{2}-1\right)^{3} \sqrt{n_{0}^{2}-1}} \\
& J_{5}=\frac{7 n_{0} J_{4}-3 J_{3}}{4\left(n_{0}^{2}-1\right)}=\frac{\pi\left(8 n_{0}^{4}+24 n_{0}^{2}+3\right)}{8\left(n_{0}^{2}-1\right)^{4} \sqrt{n_{0}^{2}-1}} .
\end{aligned}
$$

Substitution of the conditions (28) in eq. (25) leads to the horizontal distance corresponding to the highest point:

$$
\xi_{\pi}=\frac{5 \pi n_{0}\left(4 n_{0}^{2}+3\right)}{8\left(n_{0}^{2}-1\right)^{4} \sqrt{n_{0}^{2}-1}} .
$$

From eq. (17) the highest altitude is

$$
\eta_{\pi}=\frac{1}{2}\left[1-\left(\frac{n_{0}-1}{n_{0}+1}\right)^{4}\right]
$$

and the range becomes

$$
\xi_{f}=2 \xi_{\pi}=\frac{5 \pi n_{0}\left(4 n_{0}^{2}+3\right)}{4\left(n_{0}^{2}-1\right)^{4} \sqrt{n_{0}^{2}-1}} .
$$


By virtue of eqs. (6), (2), (8) and the last of (5), the time can be expressed as a function of the path inclination:

$$
\begin{aligned}
t= & 2\left(n_{0}-1\right)^{2} V_{i} I_{3} \\
= & \frac{\left(n_{0}-1\right) V_{i} \sin \gamma}{g\left(n_{0}+1\right)\left(n_{0}-\cos \gamma\right)}\left(\frac{1}{n_{0}-\cos \gamma}+\frac{3 n_{0}}{n_{0}^{2}-1}\right) \\
& +\frac{\left(2 n_{0}^{2}+1\right) V_{i}}{g\left(n_{0}+1\right)^{2}} \tan ^{-1}\left(\sqrt{\frac{n_{0}+1}{n_{0}-1}} \cdot \tan \frac{\gamma}{2}\right) .
\end{aligned}
$$

A pilot flies an aircraft by controlling the thrust and airfoil to generate acceleration, constant velocity or deceleration as per need. Hence the load factor being a function of lift coefficient, velocity and airfoil and as such of the structural strength of the aircraft, limitation of the variable load factor are concerns of engineering design.

The endurance can also be shown to be twice the time taken to reach the highest point and is given by

$$
\begin{aligned}
t_{f} & =2\left(n_{0}-1\right)^{2} V_{i} \int_{0}^{2 \pi} \frac{d \gamma}{\left(n_{0}-\cos \gamma\right)^{3}} \\
& =\frac{4\left(n_{0}-1\right)^{2} V_{i}}{g} \int_{0}^{\pi} \frac{d \gamma}{\left(n_{0}-\cos \gamma\right)^{3}}
\end{aligned}
$$

(By the use of the third of eq. (28))

$$
=\frac{2 V_{i}\left(2 n_{0}^{2}+1\right) \pi}{g\left(n_{0}+1\right)^{2} \sqrt{n_{0}^{2}-1}} .
$$

Equation (32), when combined with eq. (4), gives the time history of load factor to facilitate the manuevering of the aircraft.

\section{Discussion and conclusion}

The present way of manipulating the load factor also produces loop trajectory and the relevant differential equations of motion are also amenable to closed-form solutions.

Neglecting the loss of the airplane weight due to fuel consumption and on the same line as Miele's and recalling (7), (2.1) and (1) we need,

$$
C_{L}=\frac{C_{L i}}{u^{2}}=C_{L i}\left(\frac{n_{0}-\cos \gamma}{n_{0}-1}\right)^{2 / b}
$$

where $C_{L}$ and $C_{L i}$ respectively denote the instantaneous and initial lift coefficients. The maximum lift coefficient occurring at the highest point $(\gamma=\pi)$ must be less than the stalling lift coefficient $\left(C_{L S}\right)$ :

$$
C_{L}=C_{L i}\left(\frac{n_{0}+1}{n_{0}-1}\right)^{2 / b}<C_{L S} \Rightarrow \frac{n_{0}+1}{n_{0}-1}<\left(\frac{C_{L S}}{C_{L i}}\right)^{b / 2}
$$


or

$$
\left(C_{L i}\right)^{b / 2}\left(n_{0}+1\right)<\left(C_{L S}\right)^{b / 2}\left(n_{0}-1\right)
$$

or

$$
\left(C_{L S}\right)^{b / 2}+\left(C_{L i}\right)^{b / 2}<n_{0}\left\{\left(C_{L S}\right)^{b / 2}-\left(C_{L i}\right)^{b / 2}\right\}
$$

which leads to the inequality

$$
n_{0}>\frac{\left(C_{L S}\right)^{b / 2}+\left(C_{L i}\right)^{b / 2}}{\left(C_{L S}\right)^{b / 2}-\left(C_{L i}\right)^{b / 2}} .
$$

A pilot can increase the lift coefficient by increasing the angle of attack with the help of the shape of the airfoil up to a certain value called critical or stall angle of attack for which the stalling lift coefficient occurs, after which further increase in angle of attack brings about decrease in lift coefficient, i.e. the loop is possible for such a typical load factor function if this inequality holds good.

In closing, it can be emphasized that from an engineering design stand point, variable load factor for conservative path is quite admissible. To corroborate this aspect a mention may be made of the well-known phugoid trajectory along which, so as to maintain constant lift coefficient, the load factor is varied according to the law

$$
n=n_{i} u^{2}
$$

which is evolved when a pilot undertakes a flight with constant lift coefficient, where $u$ is the instantaneous velocity and $n_{i}$ the initial load factor. By combining eqs. (4) and (7) the present law of variation of the load factor can also be set forth as

$$
n=n_{0}+\left(n_{0}-1\right)(b-1) u^{-b},
$$

maintaining which a pilot can operate a part of his/her air travel.

However, if $n_{0}=0$ and $b=-2$, the present law (36) is tantamount to that associated with the phugoid trajectory with the initial load factor $n_{i}=3$; (figure 1).

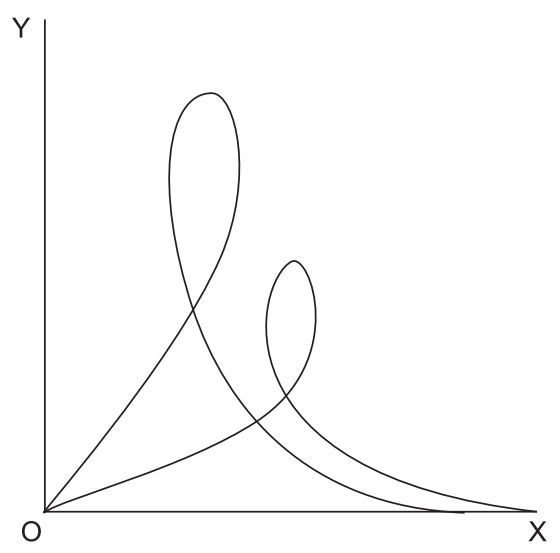

Figure 1. Phugoid trajectory of an aircraft in two flights. 
Finally, I mention that aviation authorities specify the limits of load factor with classes of aircraft to ensure flight performance without any trouble, controlling the airfoil and angle of attack.

In the foregoing analysis the variable load factor governed by relationship (4) lies between $n_{1}=b\left(n_{0}-1\right)+1$ and $n_{2}=b\left(n_{0}+1\right)-1$, due to variation of the flight path inclination from 0 to $\pi$ along the first half of the phugoid trajectory.

\section{References}

Miele A 1962 Flight Mechanics (Massachusetts: Addison - Wesley publishing company) 1: 257-273 